\title{
Relevance-based partial reliability in wireless sensor networks
}

Daniel G Costa ${ }^{1 *}$, Luiz Affonso Guedes ${ }^{2}$, Francisco Vasques ${ }^{3}$ and Paulo Portugal ${ }^{4}$

\begin{abstract}
Wireless sensor networks can provide valuable information for a lot of measurement, tracking, surveillance, automation, and general-purpose monitoring applications. Information as humidity, temperature, pressure, infrared images, and noises will be sensed and packetized for distribution over the network, but corruption during transmission may compromise the accuracy of the retrieved information and even put people in danger. In fact, the sensed data may have different relevancies for the applications, altering the impact of packet corruptions. We propose a relevance-based partially reliable transmission approach to provide data delivery with different reliability guaranties, exploiting the relevancies of transmitted data when choosing the proper error recovery service. In this context, some error scenarios are investigated, considering different configurations of error bursts. We expect that the proposed partially reliable transmission mechanism can save energy over the network while assuring acceptable quality for sensing monitoring.
\end{abstract}

Keywords: Wireless sensor networks; Partial reliability; Retransmission; Packet-level redundancy

\section{Introduction}

The development of wireless sensor network (WSN) technologies has opened new possibilities for different types of sensing applications, directly supporting monitoring in home automation, weather forecasting, traffic management, public security, industrial automation, battlefield surveillance, rescue operations, among many others $[1,2]$. In short, these networks are composed of resourceconstrained sensors that communicate through ad hoc wireless links in order to retrieve information from the monitored field. For many applications, actuators may also be employed to act in the occurrence of some events, directly benefiting automation systems.

Wireless communications over WSN are error-prone, where interferences, attenuation, and signal fading may be reflected in errors during packet transmissions. In the context of wireless sensor networks, information collected by sensor nodes may be critical for some control functions and data losses may compromise the operation of automation systems, turning reliability into a major design issue. Among the strategies for error recovery in

\footnotetext{
* Correspondence: danielgcosta@uefs.br

${ }^{1}$ Department of Technology, State University of Feira de Santana, Av

Transnordestina, s/n, Novo Horizonte, 44036-900 Feira de Santana, Brazil

Full list of author information is available at the end of the article
}

wireless sensor networks, hop-by-hop retransmission is an effective way to assure that corrupted packets will be recovered, providing full-reliable transmissions [3,4]. In fact, source nodes in WSN transmit data packets through multihop transmission paths, where each intermediate node relays packets to the next hop toward the sink. In such way, hop-by-hop retransmission approaches can assure that a new copy of any corrupted packet will be transmitted from the previous hop, significantly saving energy over the transmission path.

Packet retransmission provides reliable communications at the cost of additional energy consumption and end-to-end transmission delay. But there are other approaches for error recovery in WSN, with different outcomes. Packet-level redundancy increases the average percentage of successful packet reception at the destination when compared with unreliable transmissions, while it does not significantly compromise the transmission delay [5,6]. In a different way, when redundancy is implemented adding information into data packets, correction codes are employed in different levels of complexity, where corrupted packets may be recovered processing the codes [7]. Whatever the case, retransmission approaches typically impose low computational costs, being suitable for general- 
purpose wireless sensor networks that require full-reliable transmissions.

Sensor nodes with different sensing units may be employed for a single monitoring application, composing multitier heterogeneous sensor networks [8]. Moreover, a single node may be equipped with more than one sensing unit. Typically, sensing units will provide scalar or multimedia data: scalar information represents all data that may be presented in a scale, such as temperature and humidity, while multimedia data is generally retrieved in the form of video streams, still images, or audio. In fact, modern sensor motes usually provide multiple sensing capabilities and some hardware platforms as Arduino [9] make the insertion of additional sensing units easy. Although data redundancy may be achieved in some configurations of scalar sensors, which could lead us to avoid costly reliable transmissions, some nodes may have unique perspectives or exclusively retrieve a particular data from the monitored field $[10,11]$, requiring some level of reliability. And such requirements are more stringent in critical monitoring applications, as in industrial control and hospital automation.

For this scenario, depending on the desired monitoring function (fire detection, traffic management, industrial automation, etc.), some information may be highly critical and data losses must be avoided. For example, in a fire alert system, every packet transmitting temperature information must be preserved, and corrupted data packets must be recovered. However, sensors may be transmitting complementary information that is not critical for the monitoring functions and, therefore, some packet losses may be tolerated. As an example, infrared images may be assumed as complementary information since they can be used for fire detection, but temperature is still more crucial for very fast alerting and response. Some information may also be assumed as auxiliary in the sense that applications may tolerate any level of packet losses. For the same considered fire detection system, luminosity information may be taken as auxiliary since we cannot be sure about a fire incident just considering luminosity variations, but they may indicate that something is wrong. At last, some information will be assumed as unnecessary for the considered application and should not be transmitted in order to save energy, as for example noise and pressure in fire detection systems. One should note that different types of information may be transmitted from a single sensor or from more than one source nodes and that different applications may define different levels of data relevancies.

We propose a partially reliable transmission approach where different reliability guarantees are provided for data transmissions over wireless sensor networks, according to the relevance of the sensed data for the application. In this proposed approach, packets carrying critical information will be always retransmitted if corrupted, in a hop-by-hop fashion. On the other hand, corrupted auxiliary data packets will never be recovered, and thus, they will be transmitted in an unreliable way. For complementary packets, we propose and evaluate two different semireliable error recovery mechanisms, in the sense that reliability is only partially provided. The first mechanism is based on semi-reliable hop-by-hop retransmissions, while the last one relies on packet-level redundancy for error recovery. Depending on the network configurations and statistical error occurrence, the proposed partially reliable transmission approach is expected to bring significant results in terms of energy consumption, while keeping an acceptable level of successful packet reception.

Retransmission-based error recovery in wireless sensor networks is not a novelty, and there are many papers covering this topic. Many hop-by-hop retransmission approaches have been proposed in the last years, bringing significant contributions [12-14]. However, such approaches typically provide the same level of reliability for all transmitted packets or the reliability guaranties are not so strict to be considered for critical monitoring applications. On the other hand, we propose in this paper a partially reliable transmission approach that differentiates data packets according to their relevancies for the monitoring applications, potentially saving energy over the network without significantly compromising the overall monitoring quality.

Full-reliable and unreliable transmissions are easy to provide in WSN. But there are many ways to assure some level of semi-reliable transmissions. However, as semireliability is related to partial protection of transmitted data, different results may be achieved according to how such notion of partial protection will be implemented. In order to provide an efficient mechanism for semi-reliable delivery of complementary data packets, we propose and investigate the concept of 'error zone.' An error zone is an area of the monitored field where transmission errors will be more frequent and nodes deployed in that area will transmit packets with higher probability of errors. Such error zones may happen anywhere, and they will typically depend on the characteristics of the monitored field, as external interferences. For example, some nodes may be deployed in rooms with high electromagnetic interference, which may be common in some monitoring environments as in industrial monitoring and control applications. For hop-by-hop retransmissions, the characteristics of an error zone are central when defining the way packets will be retransmitted, directly affecting the network performance. As complementary data packets will be transmitted under a semi-reliable transmission service, and thus some packets may never reach the network sink, error zones will have deeper impact on the transmission of this kind of data, pushing us to evaluate different approaches for semi-reliable error recovery. 
In general, energy savings will be more significant for higher packet error rates since some complementary and auxiliary corrupted packets will not be recovered, but critical packets are always preserved in order not to prejudice the effectiveness of the monitoring applications. Analytical energy consumption and packet reception models were designed to assess the performance of the proposed partially reliable retransmission approach.

The remainder of this work is organized as follows. Section 2 brings up some related works. The fundamental concepts are described in Section 3. Then, Section 4 describes the proposed partially reliable transmission approach. $\mathrm{Nu}-$ merical results are presented in Section 5, followed by conclusions and references.

\section{Related works}

Generally, wireless sensor networks may pose different reliability requirements. Scalar sensors used to monitor information as temperature, pressure, and humidity may be massively deployed resulting in monitoring redundancy, which implies higher resistance to packet corruptions $[1,15]$. On the other hand, camera-enabled sensor nodes will have a unique view of the monitored field, changing the notion of redundancy and consequently the error resilience [10]. Moreover, for some monitoring environments, wireless sensor networks will have to assure that critical data is successfully received at the sink [16,17]. For all these scenarios, different approaches for error recovery have been proposed in the last years, where energy efficiency is usually the most desired optimization goal.

We can roughly classify error recovery in WSN in two different approaches: packet retransmission or redundancy $[5,6]$. Retransmission of corrupted packets assures that a new copy of a corrupted dropped packet will be retransmitted in an end-to-end or hop-by-hop way, resulting in additional information being transmitted over the network when corruption occurs. On the other hand, redundancy approaches will add information in advance, either into data packets or creating replicated packets. For the first case, correction codes are employed in different levels of complexity, where corrupted packets may be recovered processing the codes. In a different way, packet-level redundancy transmits redundant packets in advance, increasing the overall probability of successful packet reception. Each of these approaches provides different levels of reliability, energy efficiency, and processing costs $[1,18]$, but retransmission is still an effective way to provide transmission with high reliability levels and with low additional complexity, at the cost of slight additional energy consumption and transmission delay.

Actually, some key aspects and promising approaches for packet retransmission in wireless sensor networks have been investigated by the academic community in the last years, considering different strategies in one or more protocol logical layers or even following a crosslayer paradigm. Some of those works directly contributed to our investigation. In [19], the authors propose hop-byhop retransmission for sensor networks, saving energy when retransmissions happen only in the link where packet corruption occurred. Retransmission is performed in [19] when an explicit NACK message is received from the next hop toward the sink. The work in [12] combines this principle of hop-by-hop retransmission with transmissions through multiple redundant paths in order to achieve higher error resilience. Similarly, in [13], the authors propose the pump slowly, fetch quickly (PSQF) approach, a transport protocol designed for hop-by-hop error recovery in WSN. The PSQF reduces the transmission rate in the occurrence of errors, allowing fast recovery of lost packets from neighbor nodes. The work in [14] proposes the combined operation of retransmission and redundancy approaches for improved reliability and overall performance. In fact, all these works provide full-reliable transmissions for WSN applications, exploiting the hop-by-hop retransmission paradigm for higher efficiency.

Other promising approaches have been proposed to assure different levels of reliability in wireless sensor networks. The in-middle data recovery paradigm is proposed in [20], where loss detection and recovery is performed considering several hops of transmission paths, instead of only neighbor hops. Such in-middle paradigm is implemented in [20] using the idea of proliferation routing, where packet-level redundancy is exploited to produce new copies of data packets in an optimal way. The idea is to provide reliability increasing the probability of successful packet reception. Among the expected benefits, packet losses that resulted from congestion do not generate a large amount of retransmissions. In a different way, some approaches try to reduce packet corruption and transmission latency selecting the most appropriate transmission paths and wireless communication parameters, as in opportunistic routing schemes [21,22].

Sometimes, the desired level of reliability may change according to the characteristics of the monitoring application and the deployed network. As an example, a reliable transport protocol for wireless sensor networks is proposed in [23], focused on the abstraction of event detection. The proposed event-to-sink reliable transport (ESRT) protocol is concerned with the successful detection of an event, even if some data packets are lost during transmission. Depending on the network condition, continuously verified at the sink side, the transmission rate of source nodes can be adjusted to provide the minimal acceptable information for the application. Data redundancy in WSN can also alleviate the demand for reliability when equivalent data packets are transmitted, and there are many approaches focused on maximizing 
redundant sensing [24]. However, redundancy of data sensing may be hard to achieve in some monitoring scenarios, as when camera-enabled sensors are deployed [25]. In a different perspective, fountain codes in wireless sensor networks can provide reliable transmissions providing some level of diversity in the transmission flow [26], for example, combining data packets.

Whatever the employed error recovery strategy is, many wireless sensor network applications will require fullreliable transmissions, especially in critical monitoring applications. For such scenarios, which may be composed of hundreds of sensors concurrently transmitting sensed data, full-reliable transmissions can satisfactorily address application reliability requirements. However, such transmissions may also deeply impact the overall energy consumption, potentially reducing the network lifetime. We believe that the reliability requirements can be loosen for noncritical data in order to achieve higher energy efficiency.

A promising approach to optimize WSN operation is to consider different relevancies for the transmitted data. And data packets with different relevancies can be transmitted under different transmission services, with optimized reliability, availability, timeliness, and security. Some of those approaches differentiate transmitted data by the potential of source nodes to provide relevant information for the applications. In [27], a methodology for source prioritization is proposed to differentiate source nodes in five groups of relevance. In such case, packets transmitted from highrelevance source nodes may be transmitted under a fullreliable service. A similar approach is discussed in [28]. On the other hand, sometimes we can prioritize the nature of the transmitted data for any packets' origins. In [29], a semi-reliable retransmission mechanism is proposed exploiting the relevance of packets' payloads, considering discrete wavelet transform (DWT) coding over image snapshots. In that approach, only DWT subbands that are highly required to reconstruct original images are transmitted under a full-reliable transmission service. In a different way, the work in [30] proposes reliability by redundancy with correction codes, optimizing transmission when the relevance of video frames for the reconstruction of the original data is considered when defining the expected level of reliability.

Partial reliability approaches based on data relevancies are very promising. Such approaches achieve a reasonable trade-off between energy consumption and monitoring quality, but the overall application quality is not severely harmed since higher relevance data is always preserved.

In this context, we propose a partially reliable transmission approach for improved energy efficiency in wireless sensor networks. The relevancies of sensed information for the application will be considered when providing the appropriate transmission service. However, in a different way of [29], the proposed approach is generic and suitable for any kind of WSN and not only wireless image sensor networks. In our previous work [31], sensed data is classified into two different levels of relevance, where fullreliable transmission is only required for critical data while the remaining data is transmitted under a semi-reliable service. In a different way, we propose herein different transmission mechanisms for three classes of data relevancies. Moreover, we define the concept of error zones and investigate the performance of semi-reliable retransmission and packet-level redundancy over different configurations. As we are providing a relevance-based partially reliable transmission service, we expect to outperform retransmission approaches based only on full-reliable data delivery, as in [12-14,19], when applications can tolerate some lower relevance packet losses for higher energy efficiency. To the best of our knowledge, such approach has not been proposed before.

\section{Fundamental concepts}

In this section, we define analytical models for energy consumption and packet errors in wireless sensor networks, which are necessary when designing the proposed partially reliable transmission approach and when assessing its expected performance.

\subsection{Energy consumption}

We assume that wireless sensor networks are composed of $P$ hop-by-hop wireless paths and $S$ source nodes (scalar or multimedia sensors). Each path $p, p=1, \ldots, P$, comprises $H_{(p)}$ intermediate nodes, where data packets flow from the source node $(h=0)$ to the sink of the network $\left(h=H_{(p)}+\right.$ 1). Every source node $s, s=1, \ldots, S$, is connected to the sink through at least one path $p$, which is defined to be steady during transmissions (without permanent node or link failures). For simplicity, the communication scenario is assumed to be contention-free, considering transmissions using protocols as time division multiple access (TDMA) or the contention-free period in IEEE 802.15.4, but still keeping it highly realistic [32,33].

In each path, $p$ will be transmitted control and data packets, where data packets will be typically small (reducing the error probability [34]) and with the same size (reducing overhead). Thus, we consider that every data packet has the same size in bits, $k$, corresponding to the entire packet (data payload and headers). If the packet header regarding all employed protocols sizes $x$ bits, $x<k$, the maximum effective payload size for every transmitted data packet is $(k-x)$ bits.

The actual energy consumption in each node depends on many factors, as the employed radio, the transmission power, and the physical and media access control (MAC) layer protocols. Extending some energy consumption models found in the literature allows us to estimate the energy consumption in wireless sensor networks, providing 
an initial verification of the proposed optimization approach. Although we have not considered some characteristics, as transmission of synchronization messages, we believe that the designed energy consumption model is still very suitable for our verifications, since we are focused on the analyses of different results among error recovery approaches, instead of precise energy consumption estimation.

For lossless wireless links, we can define $D_{(p, h)}$ as the total amount of bits to be transmitted from hop $h$ to hop $(h+1)$ in path $p$. If we assume the total amount of packets to be transmitted in path $p$ as $W_{(p)}$, then $D_{(p, h)}=W_{(p)} \times k$. The consumed energy to send and receive bits depends on the transmission power of node $h, P w t_{(p, h)}$, and the power for bit reception, $P w r_{(p, h)}$. We define $E t_{(p, h)}$ as the energy consumption in joules for packet transmission from hop $h$ to hop $(h+1)$ in path $p$ and $\operatorname{Er}_{(p, h)}$ as the energy consumption for packet reception in hop $h$ in the same path, for $t x_{(p, h)}$ as the time for transmitting 1 bit from hop $h$, as expressed in (1):

$$
\begin{aligned}
& E t_{(p, h)}=\left\{\begin{array}{c}
D_{(p, h)} \cdot P w t_{(p, h)} \cdot t x_{(p, h)} \\
0, h=H_{(p)}+1
\end{array}\right. \\
& \operatorname{Er}_{(p, h)}=\left\{\begin{array}{c}
D_{(p, h-1)} \cdot P w r_{(p, h)} \cdot t x_{(p, h)} \\
0, h=0
\end{array}\right.
\end{aligned}
$$

The values for $P w t_{(p, h)}$ and $P w r_{(p, h)}$ can be easily computed in conventional sensor motes since most of them are powered by two AA batteries $(3.3 \mathrm{~V})$ and the energy consumed to transmit 1 bit is a known characteristic depending on the desired transmission range. For example, the MICAz mote draws $17.4 \mathrm{~mA}$ when the transmission power is $0 \mathrm{dBm}(57.42 \mathrm{~mW})$ and $14 \mathrm{~mA}$ for the transmission power of $-5 \mathrm{dBm}(46.2 \mathrm{~mW})$ [35]. As another relevant example, the value of $t x_{(p, h)}$ is $4 \mu$ s for the transmission of a single bit in IEEE 802.15.4-compliant sensors equipped with the CC24200 chipset.

The radio of the sensor nodes will have to switch between at least the transmission and reception modes, considering that for each packet transmission or reception a mode switch operation is required. The resulting equations are presented in (2), assuming $P w s_{(p, h)}$ as the power for mode switching and $t s_{(p, h)}$ as the time for each switching operation:

$$
\begin{aligned}
& E t_{(p, h)}=\left\{\begin{array}{l}
D_{(p, h)} \cdot P w t_{(p, h)} \cdot t x_{(p, h)}+W_{(p)} \cdot P w s_{(p, h)} \cdot t s_{(p, h)} \\
D_{(p, h)} \cdot P w t_{(p, h)} \cdot t x_{(p, h)}, h=0 \\
0, h=H_{(p)}+1
\end{array}\right. \\
& E r_{(p, h)}=\left\{\begin{array}{l}
D_{(p, h-1)} \cdot P w r_{(p, h)} \cdot t x_{(p, h)}+W_{(p)} \cdot P w s_{(p, h)} \cdot t s_{(p, h)} \\
0, h=0 \\
D_{(p, h-1)} \cdot P w r_{(p, h)} \cdot t x_{(p, h)}, h=H_{(p)}+1
\end{array}\right.
\end{aligned}
$$

The total consumed energy in path $p, E_{(p)}$, is achieved computing the energy consumption for transmission and reception in all nodes, as expressed in (3):

$$
E_{(p)}=\sum_{h=0}^{H_{(p)}+1}\left(E t_{(p, h)}+E r_{(p, h)}\right)
$$

\subsection{Error model}

Wireless links are error-prone in the sense that part of the transmitted packets is likely to be corrupted. It may happen when the communication links experience signal attenuation, interference, and channel fading, which may be constant in some monitoring environments. In general, errors in wireless links occur as bursts and larger packets are more likely to be corrupted $[34,36]$. In such way, we consider a Gilbert/Elliot error model that defines a Markov chain with two states: 'good' $(g)$ and 'bad' (b). For simplicity, all bits are corrected in the good state, while in the bad state at least one of the bits is corrupted [34]. The values for $g$ and $b$ depend on the physical characteristics of the considered link, as interferences, and some regions may have higher values for $b$. The Gilbert/ Elliot error model is a valuable error model still considered as a reasonable solution for modeling of errors in wireless links [34,37].

Although this model refers to bit errors, an error in a single bit will corrupt a whole packet. Thus, we are concerned with the average packet error rate (PER) for transmitted packets from hop $h$ to hop $(h+1)$ in path $p$, considering the transmission of $n$ bits. We can compute the steady-state probability for good $\left(G_{(p, h)}\right)$ and bad $(B$ $(p, h)$ states [34] in the wireless link from hop $h$ to hop $(h+1)$ in path $p$, as presented in (4). The values for $g_{(p, h)}$ and $b_{(p, h)}$, respectively, are the error probability for the good and bad states, which can be inferred from each wireless link from hop $h$ to hop $(h+1)$ [34,36]:

$$
\begin{aligned}
G_{(p, h)} & =\frac{1-b_{(p, h)}}{2-\left(g_{(p, h)}+b_{(p, h)}\right)} \\
B_{(p, h)} & =\frac{1-g_{(p, h)}}{2-\left(g_{(p, h)}+b_{(p, h)}\right)}
\end{aligned}
$$

Based on these probabilities, we can compute the average PER for a packet sizing $n$ bits $\left(P n_{(p, h)}\right)$, as expressed in (5). Such formulation is obtained considering the two cases where no bit error occurs during the transmission of a packet: the channel is in good state and remains there for the entire transmission or the channel is initially in bad state but the channel changes to good state before transmission and remains in good state for the transmission of all bits [36]:

$$
P n_{(p, h)}=1-\left(G_{(p, h)} \cdot g_{(p, h)}^{n}+B_{(p, h)} \cdot\left(1-b_{(p, h)}\right) \cdot g_{(p, h)}^{(n-1)}\right)
$$


The direct effect of transmitting larger packets is a higher packet error rate. For simplicity, we define $P d_{(p, h)}$ when $n=k$.

\subsection{Error zone}

We expect that, for some applications, error bursts will be concentrated in specific areas of the monitored field. A temporary or constant source of interference may increase the average percentage of corrupted packets in an area where some intermediate nodes may be deployed. In fact, packets can be corrupted in any wireless link, but with different probabilities. Then, we define an area with higher error rates as an error zone. In fact, an error zone may be a known characteristic of the transmission paths, in the case of constant sources or interference, and the network may be designed to minimize the effects of an error zone over the performed monitoring.

An error zone may be manifested in three different ways: in the beginning, in the middle, or in the end of any transmission path $p$. The error zone is defined by two delimiters: $B$ and $E$, where $0 \leq B \leq E \leq\left(H_{(p)}+1\right)$. If $B=0$ and $E<\left(H_{(p)}+1\right)$, the error zone is defined in the beginning of the path. If $B>0$ and $E<\left(H_{(p)}+1\right)$, the error zone is defined in the middle of the path. At last, if $B>0$ and $E=\left(H_{(p)}+1\right)$, the error zone is presented in the end of the path.

The concept of error zone is relevant only for a segment of the path that is smaller than the path itself. Thus, if $B=0$ and $E=\left(H_{(p)}+1\right)$, the transmission path does not have an error zone. Additionally, we define the same if $B=E$. For simplicity, we expect that a transmission path will have no more than one error zone, without loss of generality. Figure 1 presents different configurations of error zones.

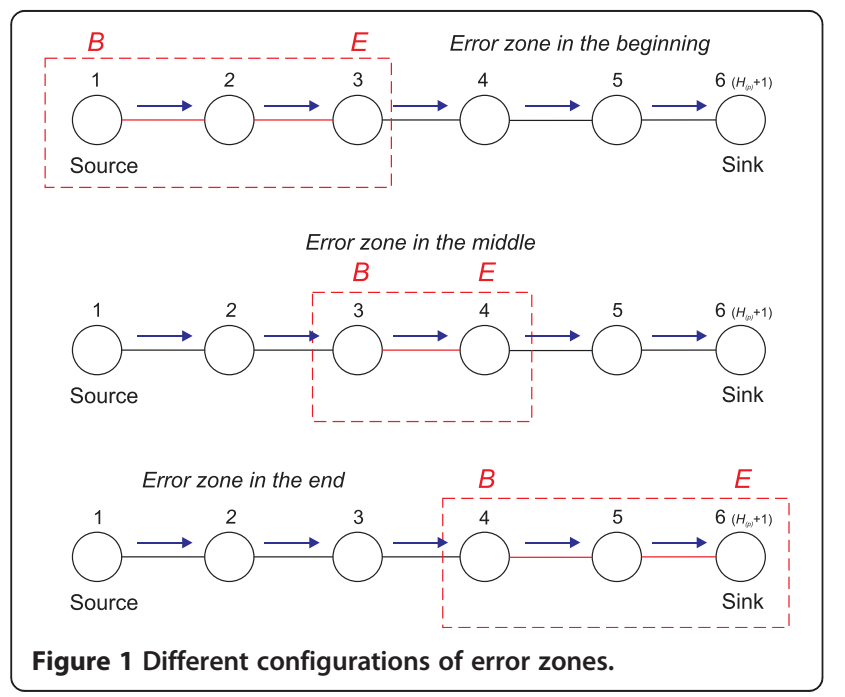

Since error zones will imply in some hops with higher packet error rates than the remaining hops of the path, an optimization mechanism may decide not to recover packets that were corrupted during transmission over an error zone. Or even the opposite could happen. In fact, different results may be achieved according to the way error recovery will be performed, as discussed in the next section.

As a final comment, the values for $B$ and $E$ may be defined deterministically before deployment or dynamically measuring the number of hops of the path and setting the best values according to the statistical occurrence of error bursts. Generally, some mechanism to indicate that a particular node is after or before $B$ or $E$ should be employed, for example, considering a marker in each data packet or including the values of $B$ and $E$ in each transmitted packet. In such case, every intermediate hop would check the corresponding values, decrementing by 1 when necessary. When that counter reaches zero, the node knows it is the delimiter and all remaining intermediate nodes in the path toward the sink will know they are after $B$ or $E$.

\section{Proposed partial reliability approach}

We propose a generic relevance-based partially reliable transmission approach for wireless sensor networks, where data packets with different relevancies are transmitted with different reliability guarantees. Data packets are classified into three different classes, where each one is associated to a particular transmission service. Next subsections present the fundaments of the proposed approach.

\subsection{Data relevancies}

Data packets are classified into three different groups, according to the relevance of the packets' payloads for the monitoring functions of the applications: critical, complementary, and auxiliary. Critical data are the most relevant and highly necessary, while auxiliary data are the least relevant for the applications. In the middle of them are complementary data that are relevant but applications can tolerate some packet losses. In fact, we expect that most source nodes will transmit complementary data in typical wireless sensor networks and only a few sensors will be critical. Moreover, we expect that transmission of auxiliary data is not mandatory and some WSN may not have this kind of data relevance. Finally, we could also define a fourth relevance class, irrelevant, that does not result in packet transmissions, but such class would have no impact in our analyses.

Critical data may be any type of sensed information, whatever is the size of data packets or requirements for timeliness. And, of course, a data type may be critical for some applications, but auxiliary for others. Moreover, control packets transmitted by the sink, as for example 
measurement requests, will be typically transmitted as critical data due to reliability requirements.

Active source nodes must properly classify each data packet before transmission. In this point, two important aspects must be remarked. First, some approach must be used to indicate the relevance of data packets, in order to allow the network to provide the expected transmission service. A reasonable approach for that is making every transmitted packet to use a special 2-bit field to be included into the packets' headers. Such data relevance (DR) field would incur in very small additional overhead in most cases, and adding control information into data packets is already a common approach in wireless sensor networks $[11,35]$. Second, source nodes must know the relevance of the information that is being transmitted by them. In fact, the relevance of the transmitted information depends on the application monitoring requirements, and thus, sensors may be previously configured in initial stages of the network lifetime. Other possibility is the use of dynamical mechanisms to compute the relevancies associated to the transmission flows, according to the interpretation of the packets received by the sink. As the monitoring requirements may change along the network operation, so may data relevancies. For such cases, centralized mechanisms as proposed in [27] could be employed.

Each level of data relevance will be associated to a transmission service, as presented in Table 1.

Packets carrying critical data will always be retransmitted if corrupted. Complementary packets may be recovered depending on the considered configurations, and we evaluated different error recovery mechanisms. At last, auxiliary packets will never be recovered if corrupted during transmission, following the formulations expressed by (2) and (3). Figure 2 presents the general schema for the proposed relevance-based partial reliability approach.

\subsection{Transmission of critical data}

As defined before, critical data must be transmitted under a full-reliable transmission service. If packets get corrupted, they will be retransmitted, where retransmission is performed in a hop-by-hop way. Thus, every transmitted packet must be successfully acknowledged by the next hop toward the sink. For simplicity and considering the use of contention-free MAC protocols, we assume that every transmitted packet on a link will be acknowledged by a 1-hop acknowledgment (ACK) message. Figure 3

Table 1 Data relevance

\begin{tabular}{lcc}
\hline Data relevance & DR & Transmission service \\
\hline Critical & 2 & Full-reliable \\
Complementary & 1 & Semi-reliable \\
Auxiliary & 0 & Unreliable \\
\hline
\end{tabular}

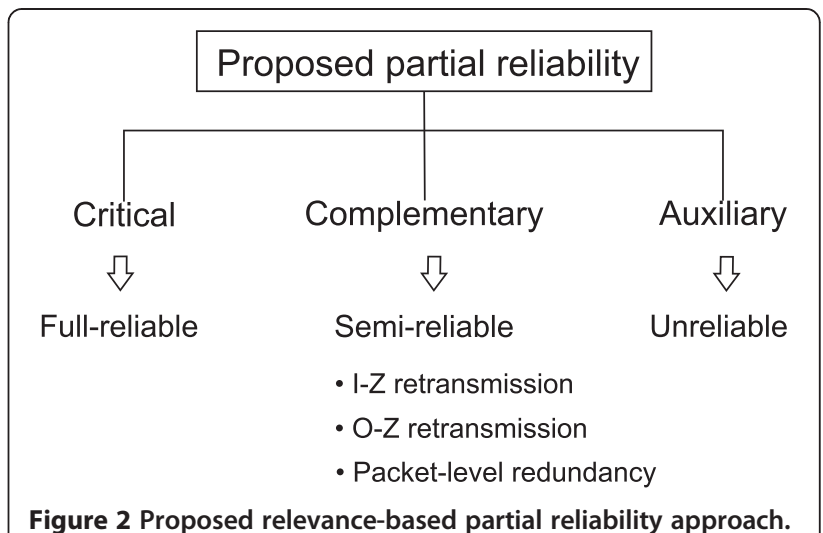

shows an example of such automatic repeat request (ARQ) in a single-path transmission.

Intermediate nodes will transmit (relay) packets following a first in, first out (FIFO) transmission queue, and critical packets at the top of the queue are only removed if they are successfully acknowledged by the next hop. Retransmissions will be triggered when ACK reception timeouts, indicating that a data packet was not properly acknowledged. Actually, this principle is generic and could be enhanced for higher efficiency, using, for example, block ACK messages.

Defining the size of ACK messages in bits as $a$ and $A$ $(p, h)$ as the total amount of bits to be transmitted for acknowledgement from hop $h$ to hop $(h-1)$ in path $p$, and $A_{(p, h)}=W_{(p)} \times a$, we achieve new formulations for energy consumption, as expressed in (6). These equations are derived from (2), just considering the additional information for transmission of ACK messages and additional state switching operations. As a comment, one should notice that source nodes $(h=0)$ do not transmit ACK messages and the sink $\left(h=H_{(p)}+1\right)$ does not receive ACKs:

$$
\begin{aligned}
& E t_{(p, h)}=\left\{\begin{array}{l}
\left(D_{(p, h)}+A_{(p, h)} \cdot P w t_{(p, h)} \cdot t x_{(p, h)}+2 \cdot W_{(p)} \cdot P w s_{(p, h)} \cdot t s_{(p, h)}\right. \\
D_{(p, h)} \cdot P w t_{(p, h)} \cdot t x_{(p, h)}+W_{(p)} \cdot P w s_{(p, h)} \cdot t s_{(p, h)}, h=0 \\
A_{(p, h)} \cdot P w t_{(p, h)} \cdot t x_{(p, h)}+W_{(p)} \cdot P w s_{(p, h)} \cdot t s_{(p, h)}, h=H_{(p)}+1
\end{array}\right\} \\
& E r_{(p, h)}=\left\{\begin{array}{l}
\left(D_{(p, h-1)}+A_{(p, h+1)}\right) \cdot P w r_{(p, h)} \cdot t x_{(p, h)}+2 \cdot W_{(p)} \cdot P w s_{(p, h)} \cdot t s_{(p, h)} \\
A_{(p, h+1)} \cdot P w r_{(p, h)} \cdot t x_{(p, h)}+W_{(p)} \cdot P w s_{(p, h)} \cdot t t_{(p, h)}, h=0 \\
D_{(p, h-1)} \cdot P w r_{(p, h)} \cdot t x_{(p, h)}+W_{(p)} \cdot \operatorname{Pws} s_{(p, h)} \cdot t s_{(p, h)}, h=H_{(p)}+1
\end{array}\right\}
\end{aligned}
$$

Packets must be retransmitted when they are corrupted in transmission from hop $h$ in path $p$ with probability $P d_{(p, h)}$. Moreover, packets must be retransmitted

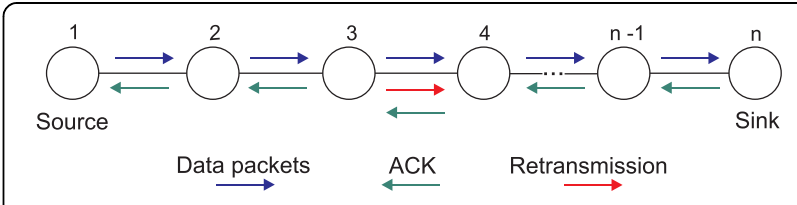

Figure 3 Hop-by-hop packet retransmission. 
when they are successfully received $\left(1-P d_{(p, h)}\right)$ but the correspondent ACK message is lost with probability $\mathrm{Pa}$ $(p, h)$, resulting in a probability of retransmission equal to $\left(\left(1-P d_{(p, h)}\right) \times P a_{(p, h)}\right)$ [34]. Considering that we could establish a maximum number of retransmission attempts as $r$, the average number of packet transmissions in the steady-state (initial transmission and retransmission attempts) of a single packet in any hop $h$ is defined in (7) [34-36]. Note that we are summing 1 to the value of $R d$ $(p, h)$ because we need to account the initial packet transmission for every data packet:

$$
\begin{aligned}
& R d_{(p, h)}=1+\left(P d_{(p, h)}+\left(1-P d_{(p, h)}\right) \cdot P a_{(p, h)}\right)+ \\
& +\left(P d_{(p, h)}+\left(1-P d_{(p, h)}\right) \cdot P a_{(p, h)}\right)^{2}+\cdots+ \\
& +\left(P d_{(p, h)}+\left(1-P d_{(p, h)}\right) \cdot P a_{(p, h)}\right)^{r}
\end{aligned}
$$

Following the formulation in (7), we can also compute the average number of ACK messages to be transmitted, as expressed in (8). The probability to send exactly one ACK message is $\left(1-P d_{(p, h)}\right)$, referring to the correct reception of a data packet. Moreover, errors in the packet transmission or in the ACK message, with subsequent retransmission, will also incur in new transmissions of ACK messages:

$$
\begin{aligned}
& R a_{(p, h)}=\left(1-P d_{(p, h)}\right)+ \\
& +\left(1-P d_{(p, h)}\right) \cdot\left(P d_{(p, h)}+\left(1-P d_{(p, h)}\right) \cdot P a_{(p, h)}\right)+ \\
& +\left(1-P d_{(p, h)}\right) \cdot\left(P d_{(p, h)}+\left(1-P d_{(p, h)}\right) \cdot P a_{(p, h)}\right)^{2}+ \\
& +\cdots+\left(1-P d_{(p, h)}\right) \cdot\left(P d_{(p, h)}+\left(1-P d_{(p, h)}\right) \cdot P a_{(p, h)}\right)^{r}
\end{aligned}
$$

Some MAC protocols may limit the value of $r$, for example, establishing $r=4$. However, for a full-reliable transmission approach, we may expect that $r$ will be very large or even virtually unlimited. In such way, the formulations in (7) and (8) become a geometric series than can be simplified, as expressed in (9) and (10):

$$
\begin{aligned}
& R d_{(p, h)}=\frac{1}{1-\left(P d_{(p, h)}+\left(1-P d_{(p, h)}\right) \cdot P a_{(p, h)}\right)} \\
& R a_{(p, h)}=\frac{\left(1-P d_{(p, h)}\right)}{1-\left(P d_{(p, h)}+\left(1-P d_{(p, h)}\right) \cdot P a_{(p, h)}\right)}
\end{aligned}
$$

Based on (9) and (10), a new formulation for the total number of bits to be transmitted is defined, as presented in (11):

$$
\begin{aligned}
& D_{(p, h)}=W_{(p)} \cdot k \cdot R d_{(p, h)} \\
& A_{(p, h)}=W_{(p)} \cdot a \cdot R a_{(p, h)}
\end{aligned}
$$

The average number of packet transmissions and retransmissions $\left(R d_{(p, h)}\right.$ and $\left.R a_{(p, h)}\right)$ will also affect the number of mode switching operations in (6), in the same way as [31]. Together, all these equations define the average theoretical energy consumption for full-reliable transmissions, which is expected for transmissions of critical data.

\subsection{Transmission of complementary data}

Complementary data will be transmitted under a semireliable service, indicating that not every transmitted packet will be protected. In the proposed approach, corrupted data packets may be recovered depending on where error bursts are occurring. We define two different approaches for semi-reliable error recovery: retransmission and packetlevel redundancy. For both mechanisms, the characteristics of error zones are central, and thus, we evaluated different strategies for packet recovery. For example, if an error zone manifests closer to the source of the transmission flow, is it a better option to only retransmit packets corrupted inside or outside the error zone? For example, if retransmissions are performed inside error zones, complementary packets are transmitted without reliability guarantees when they are being transmitted outside the error zone. This is why we call this a semi-reliable transmission approach, as some corrupted packets will not be recovered.

Although we may save energy while not severely prejudicing the quality of received complementary data, we want to identify the best approach for each configuration of error zones, in order to better support the design of wireless sensor networks.

\subsubsection{Semi-reliable retransmissions}

When packets cross error zones, they will be subject to high interference, potentially resulting in transmission errors. However, packets can also be corrupted during transmission in the remaining hops of the path (outside the error zone), but with lower probability. As there are three different configurations for error zones (in the beginning, in the middle, and in the end of the path) and two different possibilities for retransmission of corrupted complementary packets (inside (I-Z) and outside (O-Z) of the error zone), we achieve six formulations for the average amount of information that is transmitted over the considered path.

The average amount of data that is transmitted to the next hops toward the sink decreases when packets are not recovered by retransmission, for any average PER greater than zero. On the other hand, if retransmissions are performed, the exactly same amount of data that is received by an intermediate node is relayed to the next hop. Moreover, the number of ACK messages is affected by the actual data that reaches any intermediate node. These principles are considered when computing the values for $D_{(p, h)}$ and $A$ $(p, h)$ for semi-reliable transmission, as presented in Table 2.

Each formulation considers the characteristics of the error zone and if retransmissions will be performed inside (I-Z) or outside $(\mathrm{O}-\mathrm{Z})$ of any configuration of error zones. For example, in (12), the value of $D_{(p, h)}$ remains the same for I-Z when packets are crossing the error 
Table 2 Semi-reliable transmission of complementary data packets

Approach

Semi-reliable I-Z retransmission

Semi-reliable O-Z retransmission

Semi-reliable I-Z retransmission

Semi-reliable O-Z retransmission

Values for $D_{(p, h)}$ and $A_{(p, h)}$

$B=0, E<\left(H_{(p)}+1\right)$

$D_{(p, h)}=\left\{\begin{array}{l}W_{(p)} \cdot k \cdot R d_{(p, h)}, h \leq E \\ W_{(p)} \cdot k \cdot \prod_{j=E}^{h-1}\left(1-P d_{(p, h)}\right), h>E\end{array}\right.$

$A_{(p, h)}=\left\{\begin{array}{l}W_{(p)} \cdot a \cdot R a_{(p, h)}, h \leq E \\ 0, h>E\end{array}\right.$

$D_{(p, h)}=\left\{\begin{array}{l}W_{(p)} \cdot k \cdot \prod_{j=0}^{h}\left(1-P d_{(p, h)}\right), h \leq E \\ W_{(p)} \cdot k \cdot \prod_{j=0}^{E}\left(1-P d_{(p, h)}\right) \cdot R d_{(p, h)}, h>E\end{array}\right.$

$A_{(p, h)}=\left\{\begin{array}{l}0, h \leq E \\ W_{(p)} \cdot a \cdot \prod_{j=0}^{E}\left(1-P d_{(p, h)}\right) \cdot R a_{(p, h)}, h>E\end{array}\right.$

$B>0, E<\left(H_{(p)}+1\right)$

$D_{(p, h)}=\left\{\begin{array}{l}W_{(p)} \cdot k \cdot \prod_{j=0}^{h}\left(1-P d_{(p, h)}\right), h \leq B \\ W_{(p)} \cdot k \cdot \prod_{j=0}^{B}\left(1-P d_{(p, h)}\right) \cdot R d_{(p, h)}, B<h \leq E \\ W_{(p)} \cdot k \cdot \prod_{j=0}^{B}\left(1-P d_{(p, h)}\right) \cdot \prod_{j=E}^{h-1}\left(1-P d_{(p, h)}\right), h>E\end{array}\right.$

$A_{(p, h)}=\left\{\begin{array}{l}0, h \leq B \\ W_{(p)} \cdot a_{0} \cdot R a_{(p, h)}, B<h \leq E \\ 0, h>E\end{array}\right.$

$D_{(p, h)}=\left\{\begin{array}{l}W_{(p)} \cdot k \cdot R d_{(p, h)}, h \leq B \\ W_{(p)} \cdot k \cdot \prod_{j=B}^{h-1}\left(1-P d_{(p, h)}\right), B<h \leq E \\ W_{(p)} \cdot k \cdot \prod_{j=B}^{E}\left(1-P d_{(p, h)}\right) \cdot R d_{(p, h)}, h>E\end{array}\right.$

$A_{(p, h)}=\left\{\begin{array}{l}W_{(p)} \cdot a \cdot R a_{(p, h)}, h \leq B \\ 0, B<h \leq E \\ W_{(p)} \cdot a \cdot \prod_{j=B}^{E}\left(1-P d_{(p, h)}\right) \cdot R a_{(p, h)}, h>E\end{array}\right.$

$B>0, E=\left(H_{(p)}+1\right)$

$D_{(p, h)}=\left\{\begin{array}{l}W_{(p)} \cdot k \cdot \prod_{j=0}^{h}\left(1-P d_{(p, h)}\right), h \leq B \\ W_{(p)} \cdot k \cdot \prod_{j=0}^{B}\left(1-P d_{(p, h)}\right) \cdot R d_{(p, h)}, h>B\end{array}\right.$

Semi-reliable I-Z retransmission

$$
\begin{aligned}
& A_{(p, h)}=\left\{\begin{array}{l}
0, h \leq B \\
W_{(p)} \cdot a \cdot \prod_{j=0}^{B}\left(1-P d_{(p, h)}\right) \cdot R a_{(p, h)}, h>B
\end{array}\right. \\
& D_{(p, h)}=\left\{\begin{array}{l}
W_{(p)} \cdot k \cdot R d_{(p, h)}, h \leq B \\
W_{(p)} \cdot k \cdot \prod_{j=B}^{h-1}\left(1-P d_{(p, h)}\right), h>B
\end{array}\right.
\end{aligned}
$$

Semi-reliable O-Z retransmission

$$
A_{(p, h)}=\left\{\begin{array}{l}
W_{(p)} \cdot a \cdot R a_{(p, h)}, h \leq B \\
0, h>B
\end{array}\right.
$$


zone, but potentially decreases for $h \leq E$ when retransmission is only performed outside the error zone, as expressed in (13). As packets that were being transmitted in an unreliable way may start to be retransmitted when corrupted, when they leave the error zone in (13), the correct value for the number of data packets must be considered. That is the reason why the error probability for transmissions through all hops inside the error zone is accounted, resulting in $\prod_{j=0}^{E}\left(1-P d_{(p, h)}\right)$ for $h>E$.

The same is true for the other configurations. At last, one should notice that ACK messages will only be transmitted when packets are being relayed through hops that will retransmit data packets and that the same is valid for the average number of retransmissions, $R d_{(p, h)}$ and $R a_{(p, h)}$.

Depending on where higher PER (error zones) is being experienced and the adopted strategy for error recovery (retransmissions inside or outside the error zone), we can expect different energy consumption patterns. We will assess the energy consumption for these communication scenarios in the next section.

\subsubsection{Packet-level redundancy}

Besides retransmission mechanisms, packets can be replicated to increase the probability of successful reception of the original data at the destination. As an alternative to retransmission of complementary data packets, we can send a copy of each data packet along with the original packets, for higher error resilience. In such way, we propose the transmission of an additional copy for every complementary packet as one option for semi-reliable transmissions. In this case, corrupted packets are never retransmitted if corrupted. Each copy carries a clone of the original packet's payload, and the sink must consider only one copy of the received packets.

As retransmission will not be performed, $A_{(p, h)}=0$ for all nodes. The formulation for $D_{(p, h)}$ is presented in (18):

$$
D_{(p, h)}=2 \cdot W_{(p)} \cdot k \cdot\left(\prod_{j=0}^{h}\left(1-P d_{(p, h)}\right)\right)
$$

The proposed mechanism is indeed very simple but assures high probability of successful packet reception while reduces complexity avoiding retransmissions. However, in order to increase resistance for error bursts, replicated data packets should be transmitted in a scrambled order.

We will assess the theoretical performance concerning energy consumption and successful packet reception for this proposed mechanism when compared with $\mathrm{I}-\mathrm{Z}$ and $\mathrm{O}-\mathrm{Z}$ retransmissions, in order to identify the best approach for transmission of complementary data.

\subsection{Expected quality of transmitted packets}

Besides energy consumption, we can also assess the expected average success ratio for the transmitted packets. Doing so, we can compare different approaches not only concerning energy efficiency but also assessing the expect impact on the monitoring quality.

Analyses based on quality of experience (QoE) measurements are complex but can give valuable information about the monitoring quality [38]. However, such analyses are dependent of the characteristics of a particular application. As we wish to provide a generic way to evaluate monitoring quality, we formulated the average probability of successful packet reception at the destination, as presented in Table 3.

\section{Numerical results}

The average energy consumption for the proposed partial reliability approach can be estimated for different configurations of error zones in typical wireless sensor networks. We used MATLAB and the defined energy consumption equations to assess the theoretical performance of the proposed error recovery mechanisms. For all experiments, we define $k=133$ bytes, $a=40$ bytes, $x=33$ bytes, $P w t_{(p, h)}=$ $57.42 \mathrm{~mW}(0 \mathrm{dBm}), P w r_{(p, h)}=62 \mathrm{~mW}, t x_{(p, h)}=4 \mu \mathrm{s}, P w s_{(p}$ $h)=62 \mathrm{~mW}$, and $t s_{(p, h)}=10 \mu \mathrm{s}$ for all intermediate nodes, but the developed energy consumption model is also suitable for heterogeneous networks.

Initially, we estimated the energy consumption for different transmission configurations: full-reliable, semireliable with $\mathrm{I}-\mathrm{Z}$ retransmission, semi-reliable with $\mathrm{O}-\mathrm{Z}$ retransmission, semi-reliable with redundancy ('semi-reliable red'), and unreliable transmission. For this initial experiment, we assume that a single source node will be transmitting five data packets every second and we take the results for all possible transmission configurations (transmission of critical, complementary, and auxiliary data). We evaluated the energy consumption for a transmission path composed of ten intermediate nodes with an average PER of 5\%, while the error zone has an average PER of $15 \%$.

Figure 4 presents the energy consumption for an error zone defined by $B=0$ and $E=4$, considering continuous transmissions from a unique source node that is assuming different transmission configurations.

The initial obvious conclusion is that energy consumption increases when there are more packets to transmit, as expected from our energy consumption model. The unreliable transmission for auxiliary data consumes less energy in average since packets are not recovered when corrupted, in a different way of full-reliable transmission (critical data). For transmission of complementary data, there are three different possibilities: packet-level redundancy, retransmission only inside the error zone (I-Z), and retransmission only outside the error zone $(\mathrm{O}-\mathrm{Z})$. 
For the $\mathrm{O}-\mathrm{Z}$ retransmission mechanism, packets are not retransmitted if they are corrupted for $h \leq 4$, reducing the amount of information that will be reliably transmitted for $h>4$. In such way, for this scenario, $\mathrm{O}-\mathrm{Z}$ retransmission is more energy-efficient than I-Z retransmission.

Figure 5 presents the energy consumption for an error zone in the middle of the path, assuming $B=3$ and $E=7$.

A different configuration of error zone presented different results for energy consumption. Although the difference between the two retransmission mechanisms for complementary data is shorter, the O-Z retransmission is still the best approach in terms of energy consumption. Moreover, error recovery based on redundancy brought the worst results for complementary data.

We also assessed the energy consumption for an error zone in the end of the path, for $B=6$ and $E=11$, as presented in Figure 6.

Error bursts closer to the sink will be more severe in terms of energy consumption for $\mathrm{O}-\mathrm{Z}$ retransmission. For $\mathrm{I}-\mathrm{Z}$ retransmission, complementary packets will be dropped earlier and fewer packets will have to cross the remaining hops. In other words, as reliability will be provided only inside the error zone, fewer packets will be reliably transmitted through nodes closer to the sink for I-Z retransmission. We can see that behavior in Figure 6, where I-Z retransmissions were more energy-efficient in average.

We also assessed the energy consumption for an error zone with different sizes, as presented in Figure 7. We

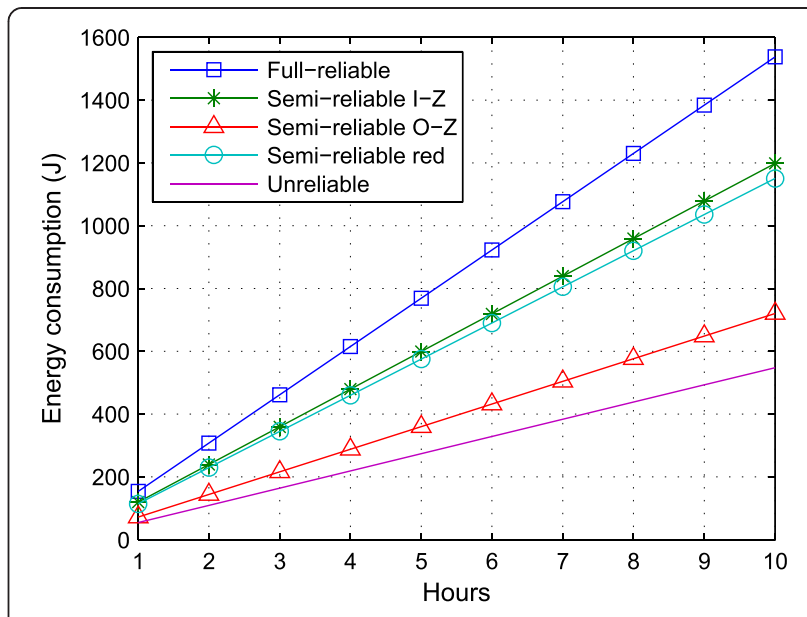

Figure 4 Energy consumption for $B=0$ and $E=4$.

assume transmissions for $10 \mathrm{~h}$ and a fixed transmission path composed of ten hops. Also, $B=0$ for this experiment.

The final energy consumption depends on the way corrupted packets will be recovered. For bigger error zones, more packets will be corrupted in average, increasing energy consumption for full-reliable transmissions and semi-reliable I-Z retransmissions. However, for $\mathrm{O}-\mathrm{Z}$ retransmissions, fewer packets will be transmitted under a reliable service when the packet error rate increases.

Table 3 Probability of successful packet reception

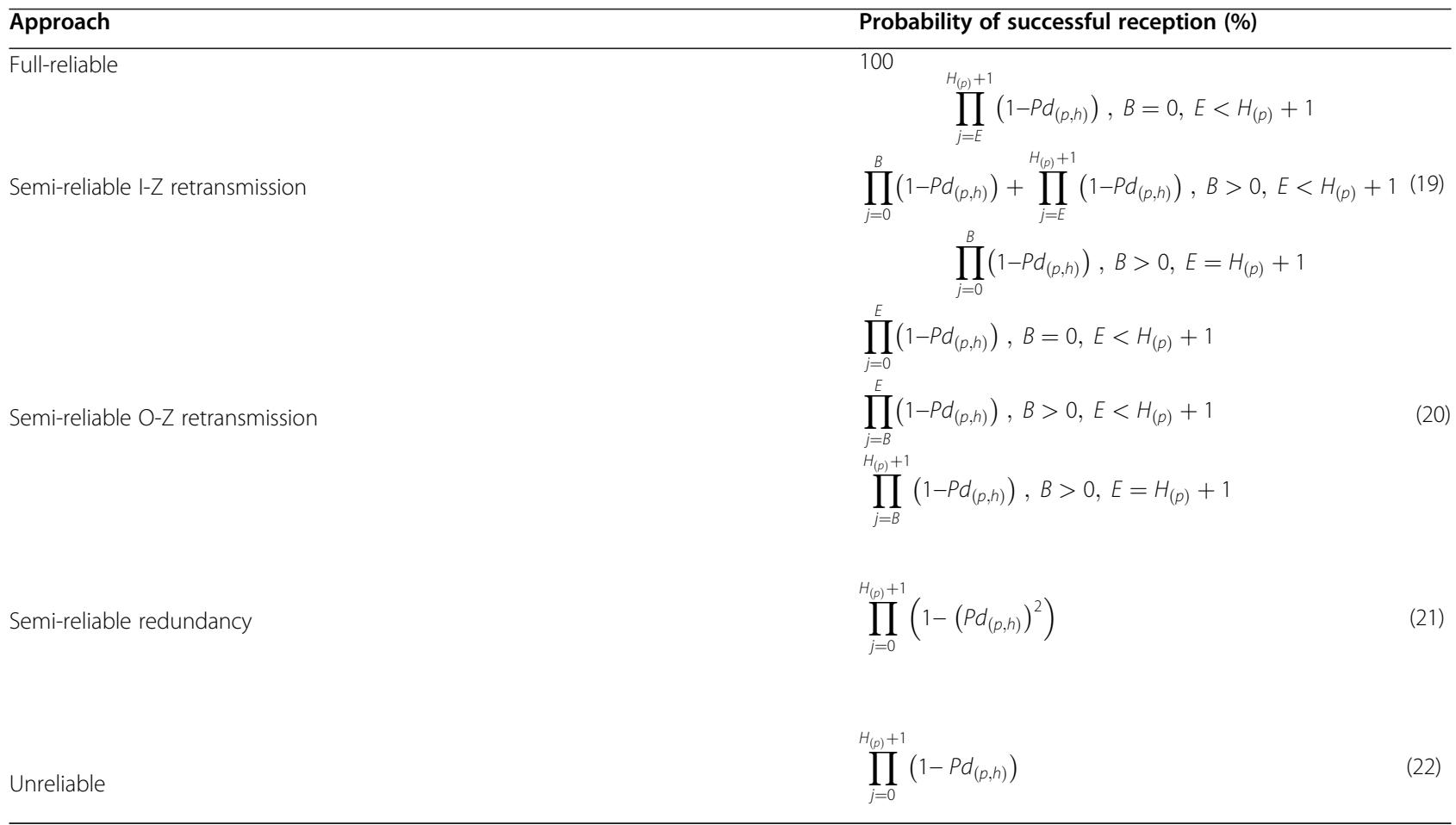




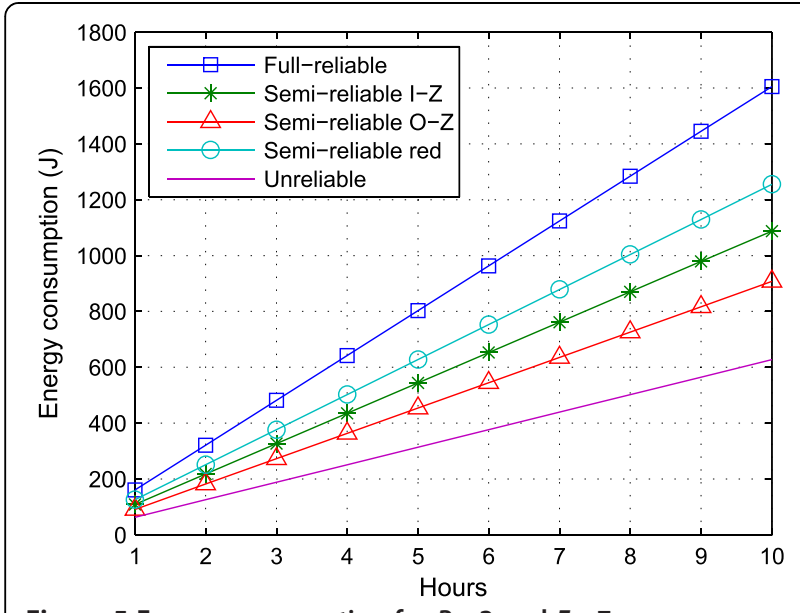

Figure 5 Energy consumption for $B=3$ and $E=7$.

The relation between PER and energy consumption was also assessed, as presented in Figure 8. We consider a path composed of ten intermediate hops, $B=3$ and $E=7$, keeping an average PER of $5 \%$ for parts of the path that are outside the error zone. The PER in the error zone is gradually increased in this experiment.

Full-reliable transmissions and semi-reliable I-Z retransmissions are more sensitive to higher PER for the error zone. Higher PER will result in more retransmissions, and as we were considering an error zone in the middle of the path $(B=3$ and $E=7)$, the energy consumption for these approaches increases. On the other hand, O-Z retransmissions will only consider error recovery outside the error zone, reducing the average energy consumption when packets enter the error zone. Unreliable transmissions and semi-reliable packet-level redundancy are also sensitive to higher PER.

Actually, the energy efficiency of the proposed error recovery mechanisms will depend on the network and error zone configurations. However, the average percentage of

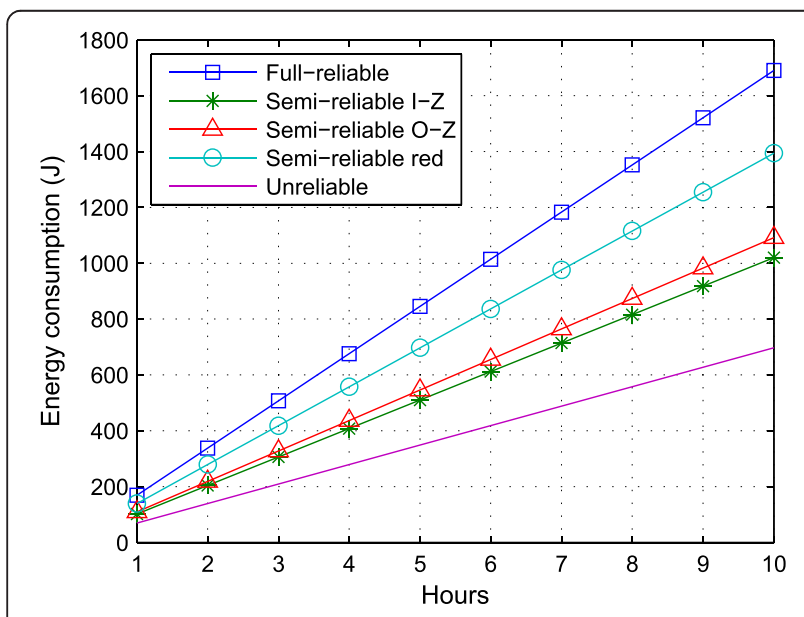

Figure 6 Energy consumption for $B=6$ and $E=11$.

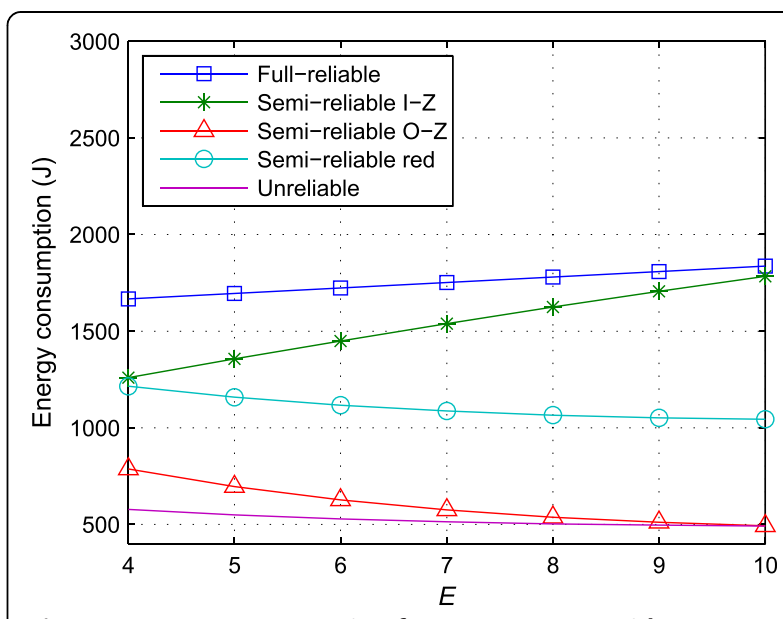

Figure 7 Energy consumption for an error zone with different sizes.

successful packet reception should be considered when assessing the performance of the proposed mechanisms. We assessed the expected probability of successful packet reception for a transmission path composed of ten intermediate hops, an error zone with PER of $15 \%$, for $B=3$ and $E=8$, and a PER of $5 \%$ for the remaining parts of the path. The results for this experiment are presented in Figure 9.

For an error zone in the middle of the path, transmissions of critical data and complementary data with retransmissions inside the error zone (I-Z) are not impacted by higher PER in the error zone. However, the other configurations are strongly impacted.

Figure 10 presents the probability of successful packet reception for $B=0$ and $E=3$.

The presented results are valid when evaluating the impact of the proposed partially reliable transmission approach. For the case of complementary data, three different approaches were proposed, each one with different expected results. In order to better highlight the performance

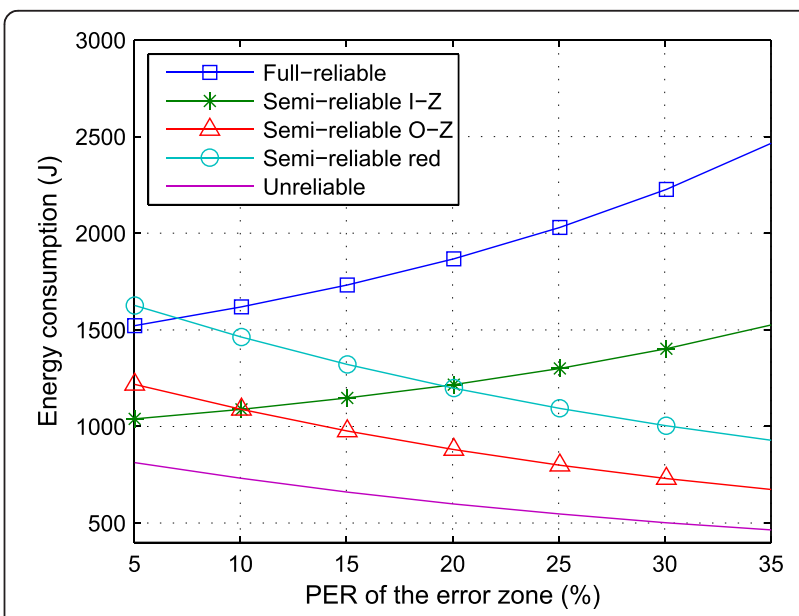

Figure 8 Energy consumption for an error zone with varying PER. 


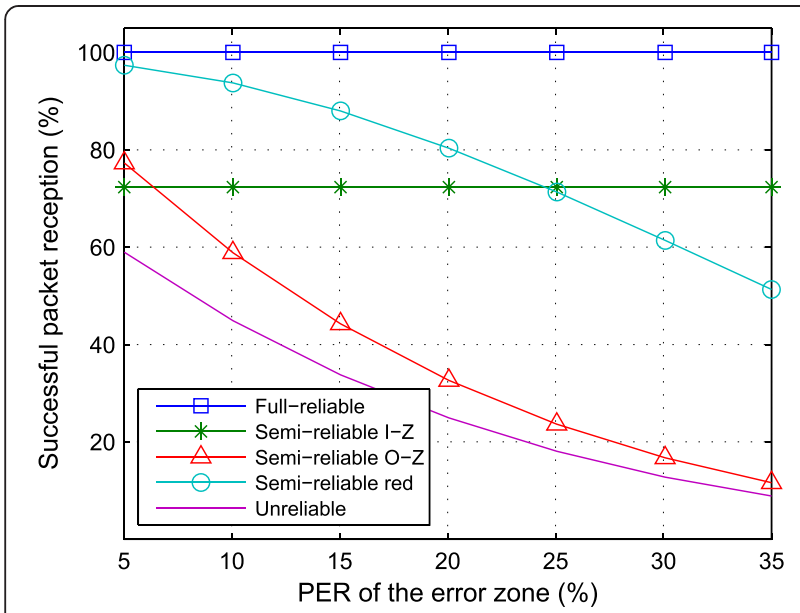

Figure 9 Probability of successful packet reception for $B=3$ and $E=8$.

of each of these three approaches, Figure 11 presents some results for transmission of only complementary data, for $10 \mathrm{~h}$ of data transmissions. We consider transmissions from a single source node that transmit five data packets every second and that is connected to the sink through ten intermediate hops. There is an average PER of $15 \%$ for the error zone and a PER of $5 \%$ for the remaining wireless links of the considered path.

As said before, the best approach for transmission of complementary data depends on the expected PER for the error zone and for remaining wireless links. Considering the performed theoretical verifications, semi-reliable transmission based on packet-level redundancy presented satisfactory results, with an average packet reception rate higher than $80 \%$. However, high energy consumption was verified for packet-level redundancy in some tests. As processing and memory costs should also be considered, future works

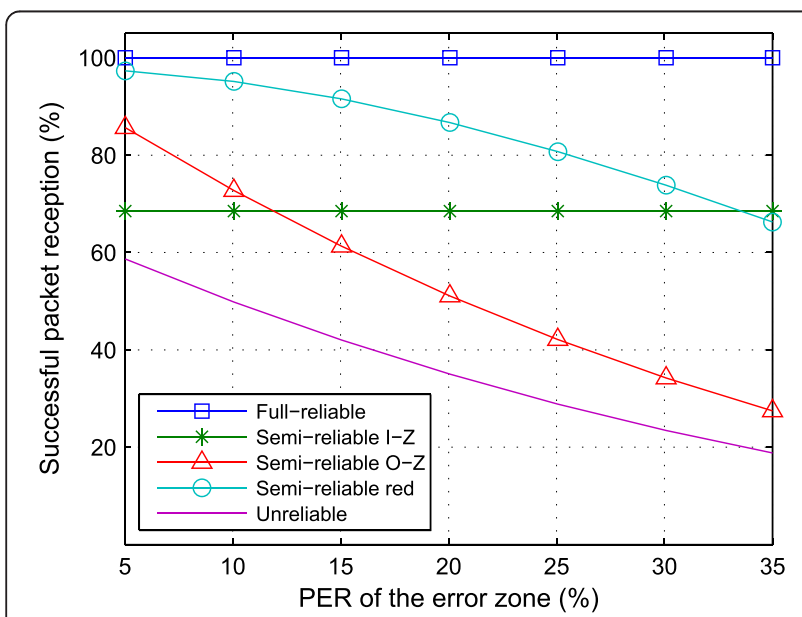

Figure 10 Probability of successful packet reception for $B=0$ and $E=3$. will be related to assessment of the overall costs for each of the three proposed semi-reliable transmission approaches.

We also investigated the expected performance of the proposed partial reliability approach for a specific WSN scenario. The communication scenario defined in Figure 12 is composed of six source nodes, each one retrieving a single type of data that has a particular relevance for the monitoring application. In that scenario, an error zone is manifested closer to the sink of the network.

Figure 13 presents the energy consumption over the communication scenario described in Figure 12. We assume an error zone with $20 \%$ of average PER and a PER of $4 \%$ for the remaining network. Moreover, for comparison purposes, we define a 'traditional' approach as full-reliable transmissions for all source nodes.

As expected, energy consumption when employing the traditional transmission approach is the highest since all packets are transmitted in a full-reliable way. For the proposed approaches, we achieve significant energy savings, according to the way corrupted complementary packets are recovered. For the considered communication scenario, where an error zone is presented in the end of the paths, I-Z retransmissions for complementary packets ('proposed I-Z') had the best results in terms of energy consumption, while error recovery by packet-level redundancy ('proposed red') presented the highest energy consumption among the proposed approaches.

Actually, the effective energy consumption depends on many factors, as the average PER of error zones and the relevance of the data that is being transmitted by the active source nodes. Moreover, where error zones are manifesting will directly impact the energy consumption when recovery of complementary packets is considered. In such way, the proper choosing of the best approach for recovery of corrupted complementary packets is a relevant design issue, and the proposed formulations for energy consumption and for successful packet reception will play an important role in that decision.

After the experiments, we expect that retransmission will be a good option for recovery of corrupted complementary packets in many scenarios, especially when energy efficiency is a major concern. In such cases, we expect that $\mathrm{O}-\mathrm{Z}$ retransmissions will be more energyefficient for error zones closer to the source, but generally I-Z retransmissions will have better results in terms of successful packet receptions. On the other hand, if applications require better average quality for complementary data, even saving less energy, error recovery by packetlevel redundancy may be the best approach.

Concerning sensing applications, we expect that the overall monitoring quality will not be severely harmed when employing the proposed approach. Critical data 

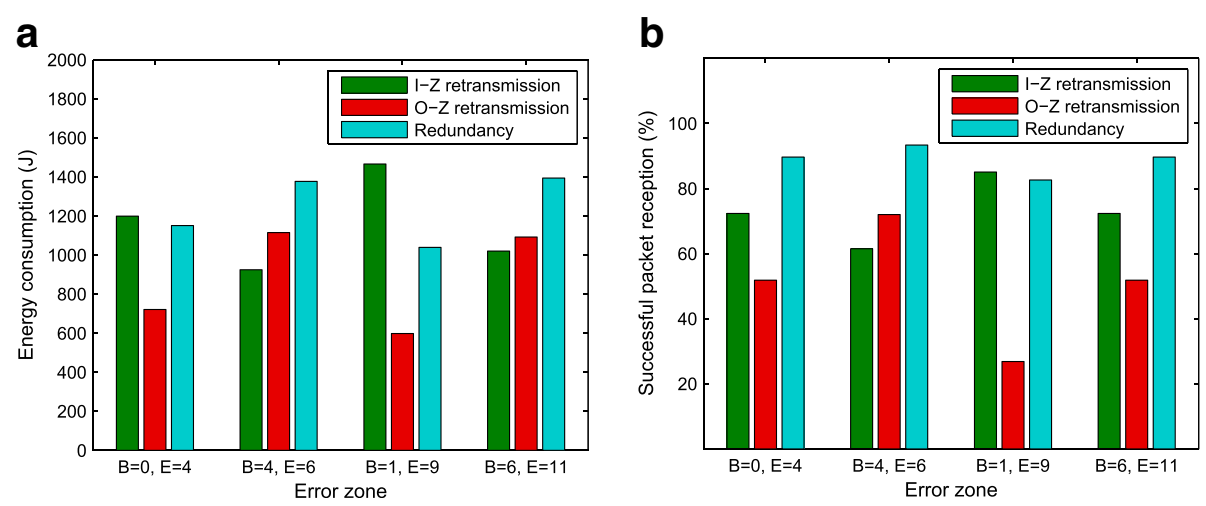

Figure 11 Expected performance for transmission of complementary data. (a) Energy consumption. (b) Packet reception.

will be always preserved and the monitoring quality will be highly dependent of this type of data. For complementary data, some packets will be lost but the actual impact on quality depends on the network configuration, the existence of error zones, and the employed error recovery strategy. And although auxiliary data packets may be significantly lost when the network experiences high PER, the overall monitoring quality is not dependent of those packets. Nevertheless, for low PER rates, most auxiliary and complementary packets will still reach the sink.

As a final comment, one should point out that auxiliary packets might not be transmitted for even higher energy efficiency. In fact, some monitoring applications might take such decision. But as the proposed partial reliability approach is aimed at generic applications, transmission of auxiliary data packets, even in an unreliable way, might be significant for many applications.

In short, the proposed relevance-based partial reliability in wireless sensor networks can significantly reduce energy consumption in WSN, which may directly benefit a lot of monitoring and control applications, as for example in industrial and mission-critical environments. Generally, we

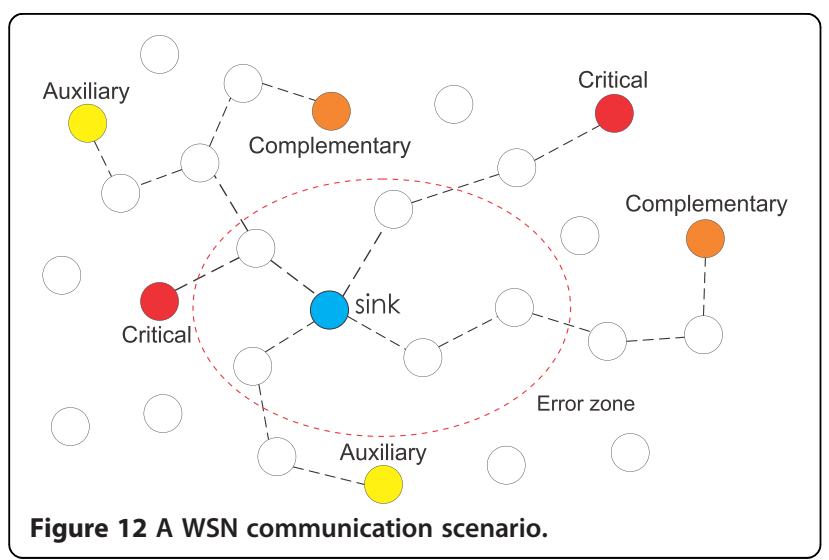

can apply the proposed transmission services in generalpurpose wireless sensor networks, whenever source nodes are transmitting data with different relevancies for the applications.

\section{Conclusions}

In this paper, we have proposed a partially reliable transmission approach where data packets are classified into one of three relevance levels according to the importance of the transmitted data for the monitoring functions of the applications. Critical data are always retransmitted if corrupted while corrupted packets carrying auxiliary data are never recovered. For complementary data, packets may be recovered by hop-by-hop retransmission or packet-level redundancy according to the employed transmission service and the presence of an error zone. We designed a comprehensive energy consumption model and performed some mathematical verification in order to attest the expected benefits of the proposed transmission approach.

Error bursts may be too severe when retransmission is enabled in resource-constrained sensor networks,

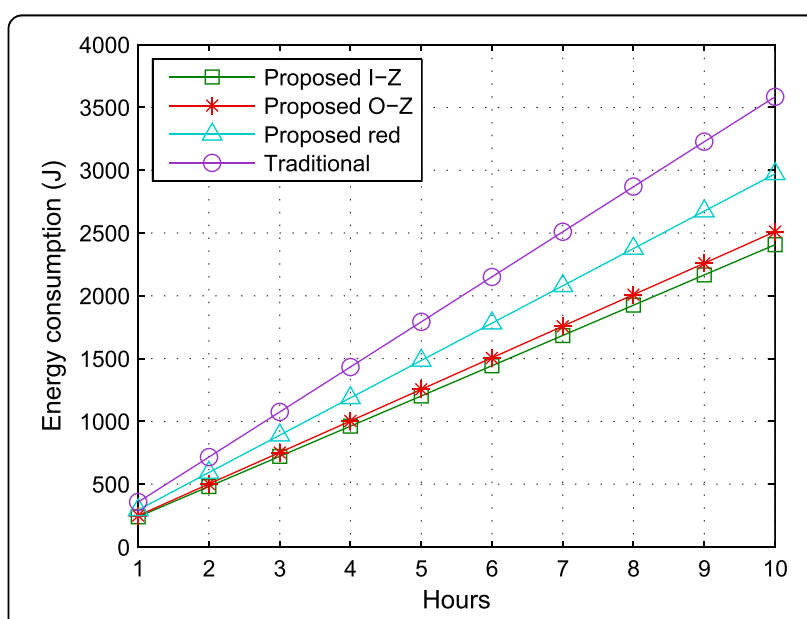

Figure 13 Energy consumption over the considered scenario. 
dramatically increasing energy consumption. Moreover, error zones may be sometimes hard to avoid and predict, and they may happen in any parts of transmission paths. For higher PER, the proposed partially reliable transmission approach will not only avoid increasing energy consumption but also provide energy savings.

As future works, the proposed approach will be extended to dynamically consider variations in the configurations of error zones. In a period of time, I-Z retransmissions may be more energy-efficient, but this configuration may change during the network lifetime, turning $\mathrm{O}-\mathrm{Z}$ retransmissions as the best approach. Adaptive networks could be able to adjust the error recovery strategy according to the current characteristics of the network. We will also investigate the impact of multiple error zones in the same sensor network. At last, we will employ a discrete-event simulator to allow more realistic analyses of punctual values of error rate, retransmission attempts, and energy consumption.

\section{Competing interests}

The authors declare that they have no competing interests.

\section{Author details}

'Department of Technology, State University of Feira de Santana, Av Transnordestina, s/n, Novo Horizonte, 44036-900 Feira de Santana, Brazil. ${ }^{2}$ Department of Computing and Automation, Federal University of Rio Grande do Norte, Campus Universitário, Lagoa Nova 59072-970 Natal, Brazil. ${ }^{3}$ IDMEC, Faculty of Engineering, University of Porto, Rua Dr. Roberto Frias, s/n 4200-465, Porto, Portugal. ${ }^{4}$ INESC TEC, Faculty of Engineering, University of Porto, Rua Dr. Roberto Frias, s/n 4200-465, Porto, Portugal.

Received: 17 November 2013 Accepted: 20 August 2014

Published: 2 September 2014

\section{References}

1. J Yick, B Mukherjee, D Ghosal, Wireless sensor network survey. Comput. Netw. 52, 2292-2330 (2008). doi:10.1016/j.comnet.2008.04.002

2. T Laukkarinen, J Suhonen, M Hännikäinen, A survey of wireless sensor network abstraction for application development. Int. J. Distrib. Sens. N. (2012). doi:10.1155/2012/740268

3. A Dunkels, J Alonso, T Voigt, H Ritter, Distributed TCP caching for wireless sensor networks, in Proceedings of Med-Hoc-Net (Bodrum, 2004)

4. H She, Z Lu, A Jantsch, D Zhou, L-R Zheng, Analytical evaluation of retransmission schemes in wireless sensor networks, in Proceedings of Vehicular Technology Conference (Barcelona, 2009)

5. H Wen, C Lin, F Ren, Y Yue, X Huang, Retransmission or redundancy: transmission reliability in wireless sensor networks, in Proceedings of IEEE MASS (Pisa, 2007)

6. M Naderi, H Rabiee, M Khansari, M Salehi, Error control for multimedia communications in wireless sensor networks: a comparative performance analysis. Ad Hoc Netw. 10, 1028-1042 (2012). doi:10.1016/j. adhoc.2012.01.003

7. W Wang, D Peng, $\mathrm{H}$ Wang, $\mathrm{H}$ Sharif, $\mathrm{H}-\mathrm{H}$ Chen, Energy-constrained distortion reduction optimization for wavelet-based coded image transmission in wireless sensor networks. IEEE Trans. Multimed. 10, 1169-1180 (2008). doi:10.1109/ TMM.2008.2001354

8. P Kulkarni, D Ganesan, P Shenoy, The case of multi-tier camera sensor networks, in Proceedings of NOSSDAV (Stevenson, 2005)

9. V Georgitzikis, O Akribopoulos, I Chatzigiannakis, Controlling physical objects via the Internet using the arduino platform over 802.15 .4 networks. IEEE Lat. Am. Trans. 10, 1686-1689 (2012). doi:10.1109/ TLA.2012.6222571

10. S Soro, W Heinzelman, On the coverage problem in video-based wireless sensor networks, in Proceedings of 2nd International Conference on Broadband Networks (Boston, 2005), pp. 932-939
11. I Almalkawi, M Zapata, J Al-Karaki, J Morillo-Pozo, Wireless multimedia sensor networks: current trends and future directions. Sens. 10, 6662-6717 (2010). doi:10.3390/s100706662

12. Y Liu, H Huang, K Xu, Multi-path-based distributed TCP caching for wireless sensor networks, in Proceedings of SNPD (Qingdao, 2007)

13. C-Y Wan, A Campbell, L Krishnamurthy, PSFQ: a reliable transport protocol for wireless sensor networks, in Proceedings of ACM International Workshop on Wireless Sensor Networks and Applications (Atlanta, 2002)

14. H Wen, C Lin, F Ren, H Yang, T He, E Dutkiewicz, Joint adaptive redundancy and partial retransmission for reliable transmission in wireless sensor networks, in Proceedings of IEEE IPCCC (Austin, 2008)

15. J Ai, A Abouzeid, Coverage by directional sensors in randomly deployed wireless sensor networks. J. Comb. Optim. 11, 21-41 (2006). doi:10.1007/ s10878-006-5975-x

16. V Gungor, G Hancke, Industrial wireless sensor networks: challenges, design principles, and technical approaches. IEEE T. Ind. Electron. 56, 4258-4265 (2009). doi:10.1109/TIE.2009.2015754

17. I Silva, L Guedes, P Portugal, F Vasques, Reliability and availability evaluation of wireless sensor networks for industrial applications. Sens. 12, 806-838 (2012). doi:10.3390/s120100806

18. C Wang, K Sohraby, B Li, M Daneshmand, Y Hu, A survey of transport protocols for wireless sensor networks. IEEE Netw. 20, 34-40 (2006). doi:10.1109/MNET.2006.1637930

19. F Stann, J Heidemann, RMST: reliable data transport in sensor networks, in Proceedings of IEEE SNPA (Anchorage, 2003)

20. $Y \mathrm{Liu}, Y \mathrm{Zhu}, \mathrm{L} \mathrm{Ni}$, A reliability-oriented transmission service in wireless sensor networks, in Proceedings of IEEE MASS (Pisa, 2007)

21. Q Lampin, D Barthel, I Auge-Blum, F Valois, QoS oriented opportunistic routing protocol for wireless sensor networks, in Proceedings of IEEE/IFIP Wireless Days (Dublin, 2012)

22. $X$ Mao, S Tang, $X X u, X-Y L i, H$ Ma, Energy efficient opportunistic routing in wireless sensor networks. IEEE Trans. Parallel Distrib. Syst. 22, 1934-1942 (2011). doi:10.1109/TPDS.2011.70

23. Y Sankarasubramaniam, Ö Akan, I Akyildiz, ESRT, event-to-sink reliable transport in wireless sensor networks. IEEE/ACM Trans. Netw. 13, 1003-1016 (2005). doi:10.1109/TNET.2005.857076

24. C Huang, Y Tseng, The coverage problem in a wireless sensor network, in Proceedings of 2nd ACM WSNA (San Diego, 2003)

25. L Liu, H Ma, X Zhang, On directional k-coverage analysis of randomly deployed camera sensor networks, in Proceedings of the IEEE ICC (Beijing, 2008)

26. A Apavatjrut, C Goursaud, K Jaffrès-Runser, C Comaniciu, J-M Gorce, Toward increasing packet diversity for relaying $L T$ fountain codes in wireless sensor networks. IEEE Comm. Letters 15, 52-54 (2011). doi:10.1109/ LCOMM.2010.111910.101692

27. D Costa, $L$ Guedes, Exploiting the sensing relevancies of source nodes for optimizations in visual sensor networks. Multimed. Tools Appl. 64, 549-579 (2013). doi:10.1007/s11042-011-0961-4

28. A Sharif, V Potdar, A Rathnayaka, Priority enabled transport layer protocol for wireless sensor network, in Proceedings of IEEE AINA (Perth, 2010)

29. D Costa, L Guedes, A discrete wavelet transform (DWT)-based energy-efficient selective retransmission mechanism for wireless image sensor networks. J. Sens. Act. Netw. 1, 3-35 (2012). doi:10.3390/jsan1010003

30. Z Zhao, D Rosário, E Cerqueira, R Immich, M Curado, QoE-aware FEC mechanism for intrusion detection in multi-tier wireless multimedia sensor networks, in Proceedings of IEEE WiMob (Barcelona, 2012)

31. D Costa, L Guedes, F Vasques, P Portugal, Partial energy-efficient hop-by-hop retransmission in wireless sensor networks, in Proceedings of IEEE INDIN (Bochum, 2013)

32. $\mathrm{H}$ Wu, A Abouzeid, Error resilient image transport in wireless sensor networks. Comput. Netw. 50, 2873-2887 (2006). doi:10.1016/j. comnet.2005.09.039

33. V Lecuire, C Duran-Faundez, N Krommenacker, Energy-efficient transmission of wavelet-based images in wireless sensor networks. EURASIP J. Image Video Process. (2007). doi:10.1155/2007/47345

34. J Korhonen, Y Wang, Effect of packet size on loss rate and delay in wireless links, in Proceedings of IEEE WCNC (New Orleans, 2005)

35. D Costa, L Guedes, F Vasques, P Portugal, Effect of frame size on energy consumption in wireless image sensor networks, in Proceedings of IEEE IST (Manchester, 2012)

36. B Han, $\mathrm{S}$ Lee, Efficient packet error rate estimation in wireless networks, in Proceedings of TridentCom (Orlando, 2007) 
37. G Anastasi, M Conti, M Francesco, Extending the lifetime of wireless sensor networks through adaptive sleep. IEEE Trans. Ind. Informat. 5, 351-365 (2009). doi:10.1109/TII.2009.2025863

38. P Brooks, B Hestnes, User measures of quality of experience: why being objective and quantitative is important. IEEE Netw. 24, 8-13 (2010). doi:10.1109/MNET.2010.5430138

doi:10.1186/1687-1499-2014-142

Cite this article as: Costa et al:: Relevance-based partial reliability in

wireless sensor networks. EURASIP Journal on Wireless Communications and Networking 2014 2014:142.

Submit your manuscript to a SpringerOpen ${ }^{\circ}$ journal and benefit from:

- Convenient online submission

- Rigorous peer review

- Immediate publication on acceptance

- Open access: articles freely available online

- High visibility within the field

- Retaining the copyright to your article

Submit your next manuscript at $\gg$ springeropen.com 